\title{
A New Circumscribed Self-Excited Spherical Strange Attractor
}

\author{
Ramesh Ramamoorthy, ${ }^{1}$ Sajjad Shaukat Jamal, ${ }^{2}$ Iqtadar Hussain, ${ }^{3}$ Mahtab Mehrabbeik (D), \\ Sajad Jafari, ${ }^{4,5}$ and Karthikeyan Rajagopal $\mathbb{1 D}^{6}$
}

${ }^{1}$ Center for Artificial Intelligence, Chennai Institute of Technology, Chennai, India

${ }^{2}$ Department of Mathematics, College of Science, King Khalid University, Abha, Saudi Arabia

${ }^{3}$ Department of Mathematics, Statistics and Physics, Qatar University, Doha 2713, Qatar

${ }^{4}$ Biomedical Engineering Department, Amirkabir University of Technology, Tehran, Iran

${ }^{5}$ Health Technology Research Institute, Amirkabir University of Technology, Tehran, Iran

${ }^{6}$ Center for Nonlinear Systems, Chennai Institute of Technology, Chennai, India

Correspondence should be addressed to Karthikeyan Rajagopal; rkarthiekeyan@gmail.com

Received 27 April 2021; Accepted 4 June 2021; Published 11 June 2021

Academic Editor: Shijian Cang

Copyright (C) 2021 Ramesh Ramamoorthy et al. This is an open access article distributed under the Creative Commons Attribution License, which permits unrestricted use, distribution, and reproduction in any medium, provided the original work is properly cited.

\begin{abstract}
Studying new chaotic flows with specific characteristics has been an open-ended field of exploring nonlinear dynamics. Investigation of chaotic flows is an area of research that has been taken into consideration for many years; thus, it helps in a better understanding of the chaotic systems. In this paper, an original chaotic 3D system, which has not been investigated yet, is presented in spherical coordinates. A unique feature of the proposed system is that its velocity becomes zero for a specific value of the radius variable. Hence, the system's attractor is expected to be stuck on one side of a plane in spherical coordinates and inside or outside a sphere in the corresponding Cartesian coordinates. It means that the attractor cannot pass through the sphere or even touch it. The introduced system owns two unstable equilibria and a self-excited strange attractor. The 1D and 2D system's bifurcation diagrams concerning the alteration of two bifurcation parameters are plotted to investigate the system's dynamical properties. Moreover, the system's Lyapunov exponents in the corresponding period of bifurcation parameters are calculated. Then, two 2D basins of attraction for two different third dimension values are explored. Based on the basin of attraction, it can be found that the sphere has attraction itself, partially, and some initial conditions are led to the sphere, not to the strange attractor. Ultimately, the connecting curves of the proposed system are explored to find an informative 1D set in addition to the system's equilibria.
\end{abstract}

\section{Introduction}

It has been revealed that simple, low-dimensional, and nonlinear mathematical differential equations can lead to complex and chaotic behaviors $[1,2]$. Design or identification of unique special systems that can exhibit chaotic performance is one of the hottest topics in nonlinear mathematics [3-5]. Since the occurrence of chaotic behavior in the system's dynamic has always been taken into consideration, numerous investigations have been performed on particular characteristics of chaotic or nonlinear systems, and diverse systems have been introduced. In other words, some researchers have introduced multistable [6-9], megastable [10-12], extreme multistable [13-15], variable- boostable [16, 17], memristor-based [18-20], conservative [21-23], multicluster [24], or any kinds of symmetrical systems [25-28]. For instance, the paper proposed by Bao et al. has introduced a nonautonomous 2D neural system and has also studied the multistability of the defined model [7]. The proposed model has been found to have an AC equilibrium and also two coexisting attractors, which have been considered as different neural firing activities. Another good example of multistability has been proposed by Bayani et al. [9]. They have introduced a $4 \mathrm{D}$ multistable system. The defined system was found to possess a plane of equilibria and also a hidden attractor. Megastability is a term first coined by Sprott et al., and it refers to the systems that include an unlimited number of countable coexisting attractors [10]. 
They have also investigated a cabbage-like model by forcing the $2 \mathrm{D}$ van der Pol system and have shown that infinite nested coexisting attractors and, in general, more complicated dynamics can be presented by forcing a simple system, such as a van der Pol oscillator. A similar term to megastability is extreme multistability which refers to the system with a limitless number of uncountable coexisting attractors [29]. An illustration of an extreme multistable system has been presented by Zhang et al. [14]. They have proposed a hyper-jerk system that showed an extreme multistability property. Furthermore, a list of variable-boostable systems-the domain of which can be partially controlled by a parameter-are provided by Li and Sprott [16]. Since the design of memristor-based and fractional-order systems have been interesting fields in nonlinear dynamics, Ruan et al. have presented a simple fractional-order memristorbased system [30]. Since Li et al.believed that the conservative chaotic systems are more appropriate for information security, they have proposed a conservative 3D system with 6 clusters and also a single fixed tori [22]. Moreover, some examples of different symmetrical systems have been proposed by Li et al. [27]. They have expressed how to construct a system with conditional symmetry and then have provided some systems as examples.

The study and realization of the system's equilibria's distribution play an important role in recognizing and identifying strange attractors. It used to be believed that strange attractors were associated with saddle points. However, chaos has been found in some systems with no or one stable equilibrium $[31,32]$. Due to the attractiveness of this issue, different chaotic systems have been proposed to discover the secret of the strange attractor's presence in nonlinear systems. Thus, some studies have proposed systems with no equilibria [33-35], nonhyperbolic equilibria $[36,37]$, or stable equilibria [38, 39]. Some others include the systems with a specific configuration of equilibria such as line [40], curve [41, 42], plane [43], or surface [44]. Besides the equilibria points of a system which are zero-dimensional invariant sets, connecting curves, which are one-dimensional sets, can also provide helpful information about the trajectory of a system. Connecting curves provide more general information about the nature of flows and trajectories than local information provided by the fixed points. Connecting curves insistently pass through the fixed points. However, they are not dependent on them $[45,46]$.

Bifurcation diagram and Lyapunov exponent are the two primary tools for investigating the dynamics of a system $[47,48]$. The bifurcation diagram of a system demonstrates the system's dynamics and its alteration in proportion to the variation of a control parameter. Lyapunov exponents of a system are the main objective and quantitative measure of the presence of chaos in a system. The number of Lyapunov exponents is based on the dimension of the system. Moreover, the largest Lyapunov exponents (LLE) value can determine the system's dynamic in specified parameters. For instance, a positive value of LLE can be evidence of chaos's existence in the system's dynamics [47].

Among other topics, one of the new-brand categories of studying the nonlinear system's dynamic is hidden attractors
[49-51]. Based on the studies declared in [52-54], generally, two principal categories can be defined for the system's attractors: self-excited and hidden attractors. A self-excited attractor is a kind of attractor that possesses an unstable equilibrium within its basin of attraction. Therefore, using numerical computations, a self-excited attractor can be easily determined. It seems that the unstable equilibrium provides a mean for the self-excited attractor. In numeric solution, the trajectory is absorbed from a point near the unstable equilibrium to the oscillating attractor and traces it [55]. In contrast, the basin of attraction of a hidden attractor is equilibrium-free. Hence, hidden attractors are expected to be found in nonequilibrium systems or systems with entirely stable equilibria [56].

In the present paper, an original chaotic system with a restricted self-excited attractor is proposed using a comprehensive computational search. This system is initially defined in spherical coordinates, which is discussed in detail in Section 2. Section 3 investigates the various dynamic properties of the proposed system. The implementation of the system's connecting curves is explored in Section 4 . Finally, Section 5 provides a conclusion for the paper.

\subsection{The Spherical Definition of the New Chaotic System.} Lately, the exploration of chaotic systems in spherical coordinates has been an exciting topic [57-59]. The appeal of such systems is that their chaotic attractor in the Cartesian coordinates, like a captivated bird in a cage, is restricted to a sphere of radius $k$. In this paper, another novel bird system with the stated characteristic in the spherical coordinates is presented as follows:

$$
\begin{aligned}
& \dot{\rho}=b \rho \varphi-a \rho-10 b \varphi+10 a, \\
& \dot{\theta}=\varphi^{2}-c \theta \varphi \\
& \dot{\varphi}=\theta^{2}+\rho \theta-10 \theta
\end{aligned}
$$

Here, $\rho, \theta$, and $\varphi$ are denoted to the radial state, azimuthal state, and polar state, respectively. Also, $a, b$, and $c$ are the system's parameters. According to system (1), it can be seen that when $\rho$ variable reaches 10 , the velocity of $\rho$ variable, i.e., $\dot{\rho}$, becomes zero. In other words, as soon as the system's output reaches the $\rho=10$ plane, it permanently remains on it. Hence, the attractor of the system, whether on the left or right side of the plane $\rho=10$, cannot pass through the plane. In the spherical coordinates, the plane $\rho=10$ is corresponding to a sphere of radius 10 in the Cartesian coordinates. Besides, depending on which side of the plane $\rho=10$ of the system attractor is located in the spherical coordinates, the corresponding attractor in Cartesian coordinates is limited to inside or outside the sphere with a radius of 10 . The investigation on the dynamic of system (1) revealed that this system has a strange attractor for $a=2$, $b=1$, and $c=1$ using the initial condition of $\left(\rho_{0}, \theta_{0}, \varphi_{0}\right)=(-2,3,0)$. The initial condition is selected, using extensive computational search, in the left side of the plane $\rho=10$ or inside the sphere of radius 10 . So, the attractor of the system remains inside this sphere. Using the transformation $x=\rho \cos (\theta) \sin (\varphi), y=\rho \sin (\theta) \sin (\varphi)$, and 
$z=\rho \cos (\varphi)$, Figure 1 illustrates the three-dimensional trajectories of the proposed system in Cartesian coordinates along with its three $2 \mathrm{D}$ projections in $\mathrm{x}-\mathrm{y}, \mathrm{x}-\mathrm{z}$, and $\mathrm{y}-\mathrm{z}$ planes.

According to Figure 1, it is apparent that the strange attractor of the proposed system is captivated in a sphere of radius 10 .

\section{Dynamic Properties}

Finding the system's equilibria and analyzing their stability is the primary analysis that can be performed on a system in order to obtain a better recognition of the system. To achieve the equilibria of system (1), the velocity of each three states, i.e., $\dot{\rho}, \dot{\theta}$, and $\dot{\varphi}$ should be set to zero. So, it can be written that $b \rho \varphi-a \rho-10 b \varphi+10 a=0, \varphi^{2}-c \theta \varphi=0$, and $\varphi^{2}-c \theta \varphi=0$. From the first equation, $\rho=10$ or $\varphi=(a / 2)$. Similarly, from the second equation, $\varphi=0$ or $\varphi=c \theta$. Finally, from the third equation, $\theta=0$ or $\theta=10-\rho$. The result of the combination of these conditions leads to two equilibria which are $\left(\rho_{1}^{*}, \theta_{1}^{*}, \varphi_{1}^{*}\right)=(10,0,0)$ and $\left(\rho_{2}^{*}, \theta_{2}^{*}, \varphi_{2}^{*}\right)=(10-(a / c b),(a / c$ $b),(a / b))$. To examine the stability of computed equilibria, the Jacobian matrix of system (1) is calculated as follows:

$$
J=\left[\begin{array}{ccc}
b \varphi-a & 0 & b \rho-10 b \\
0 & -c \varphi & 2 \varphi-c \theta \\
\theta & 2 \theta+\rho-10 & 0
\end{array}\right] .
$$

For each equilibrium of the system, the signs of the real part of the characteristic equation roots determine whether the applied equilibrium is stable or not. The characteristic equation can be obtained from

$$
|\lambda I-J|=0 .
$$

Using the Jacobian matrix and equation (3), the characteristic equation for $\left(\rho_{1}^{*}, \theta_{1}^{*}, \varphi_{1}^{*}\right)=(10,0,0)$ is $\lambda^{3}+2 \lambda^{2}=0$. So, the first equilibrium $\left(\rho_{1}^{*}, \theta_{1}^{*}, \varphi_{1}^{*}\right)$ has two zero and one negative eigenvalues. Therefore, it is not possible to determine its stability using the analytic method. However, the numeric calculation shows that this equilibrium is unstable and settled on the sphere in Cartesian coordinates. In the same way, the characteristic equation for $\left(\rho_{2}^{*}, \theta_{2}^{*}, \varphi_{2}^{*}\right)=$ $(10-(a / c b),(a / c b),(a / b))$ is $\lambda^{3}+2 \lambda^{2}+8=0$, which leads to one negative real eigenvalue $\left(\lambda_{1}=-2.93\right)$ and two complex eigenvalues with positive real parts $\left(\lambda_{2,3}=\right.$ $0.46 \pm 1.58 i)$. So, the second equilibrium $\left(\rho_{2}^{*}, \theta_{2}^{*}, \varphi_{2}^{*}\right)$ is unstable as well.

The bifurcation diagram and the corresponding system's Lyapunov exponents are studied to investigate the different dynamical performances of the proposed system. Figure 2 represents the bifurcation diagram and Lyapunov exponents of system (1) corresponding to the alteration of two parameters, namely, $b$ and $c$. The bifurcation diagram of the system is plotted using the Poincaré section. More specifically, the peaks or maximum values of the $\rho$ variable are determined as the desired Poincaré section for obtaining the bifurcation diagrams. Also, the selected initial condition is considered constant for each bifurcation parameter. The Wolf algorithm [60], with a run-time of 10000, is used to obtain the system's Lyapunov exponents.

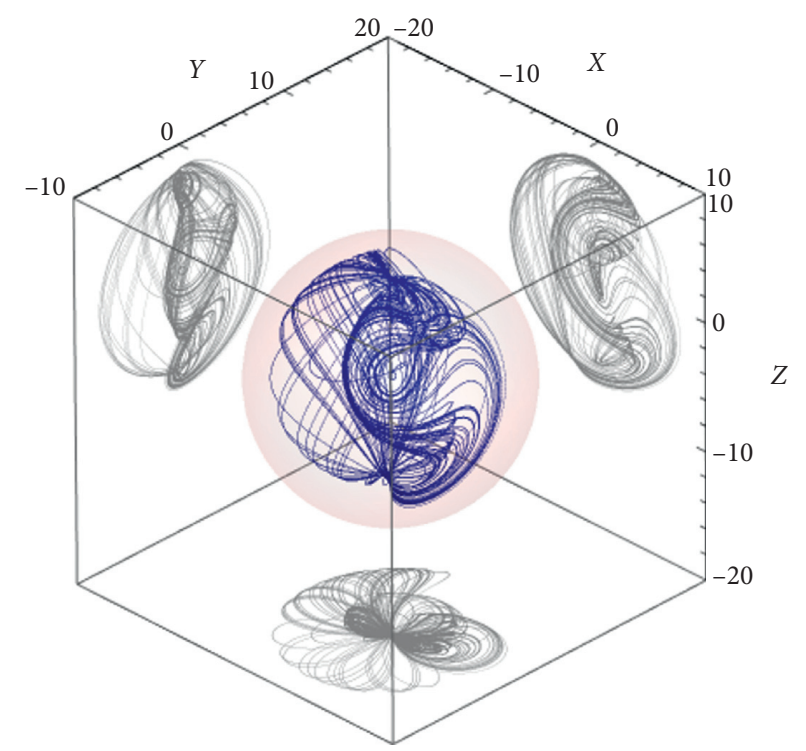

FIgure 1: The 3D plot of the strange attractor of system (1) bounded in the sphere of radius 10 (red sphere) in the Cartesian coordinates for $a=2, b=1$, and $c=1$ and initial condition of $\left(\rho_{0}, \theta_{0}, \varphi_{0}\right)=(-2,3,0)$. The three $2 \mathrm{D}$ projections in the $x-y, x-z$, and $y-z$ plane are represented in gray. The system attractor is clearly bounded to a sphere of radius 10 and cannot pass through or even touch it.

Generally, considering $a=2$ and $c=1$, Figure 2(a) shows chaotic and periodic behaviors for $b \approx[0.7,1.09]$ and $b \approx(1.09,2.5]$, respectively. Figure 2 (b) illustrates a positive, a negative, and one zero Lyapunov exponents for $a=2, c=1$, and $b \approx[0.7,1.8]$ that demonstrate the chaotic performance of the system. Moreover, assuming $a=2$ and $b=1$, Figure 2(c) shows periodic and chaotic performances for $c \approx[0.5,0.97)$ and $c \approx[0.97,1.5]$, respectively. Similarly, Figure 2(d) illustrates a positive, a negative, and one zero Lyapunov exponents for $a=2, b=1$, and $c \approx[0.97,1.5]$ that demonstrate the system's chaotic behavior. Consequently, determining $a=2$, the introduced system shows a chaotic behavior in $b=1$ and $c=1$. For $a=2$ and $c=1$, it is noticeable that, by raising the parameter $b$, the system exhibits an inverse period-doubling route to chaos. On the contrary, assuming $a=2$ and $b=1$, a period-doubling route to chaos can be discerned in the system's dynamic by raising the parameter $c$. Also, Figure 2 shows both periodic dynamics and chaotic behavior.

Figure 3 illustrates two 2D bifurcation diagrams of system (1) corresponding to the variation of parameters $b$ and $c$. Different bifurcation diagrams of the system can be observed for the simultaneous changes of parameter $b$ in different values of parameter $c$ and vice versa. Figure 3 can help to discover the system's dynamics in different parameter values at the same time.

In the study of systems with chaotic behavior, the initial conditions and the parameters' values can lead to the different behavior of the system, especially when the system is multistable. Therefore, calculating the system's basin of attraction can be an excellent method to study the sensitivity of the system's ultimate output to various initial values. So, 


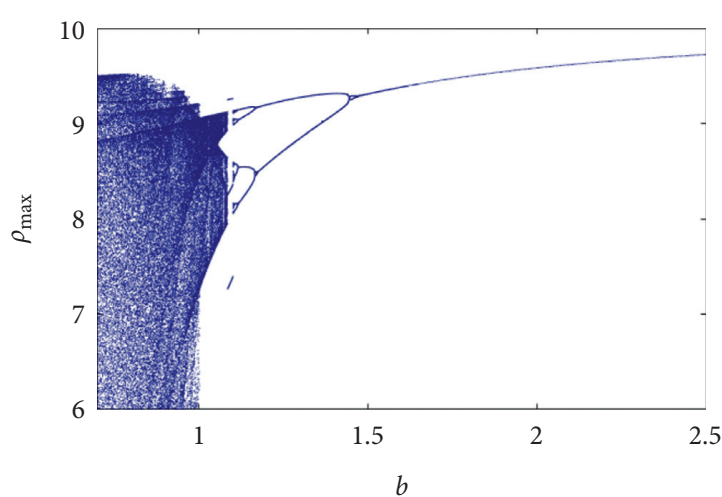

(a)

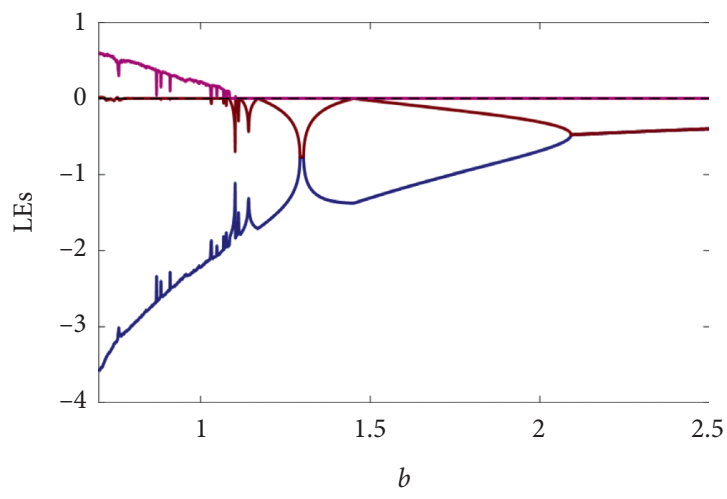

(c)

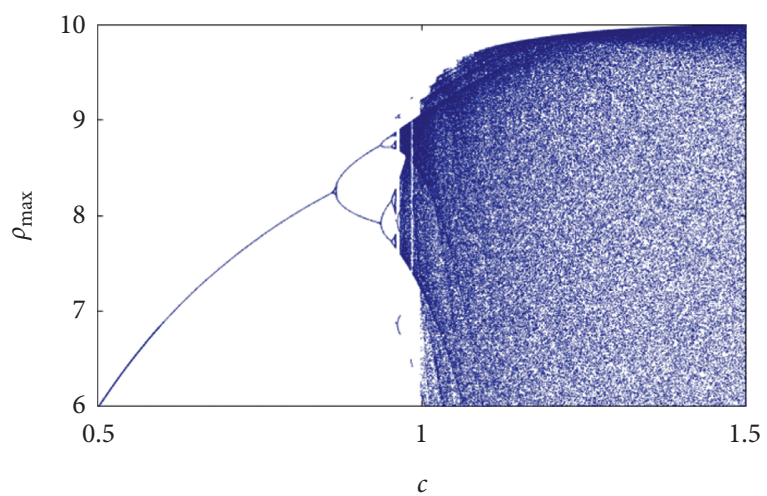

(b)

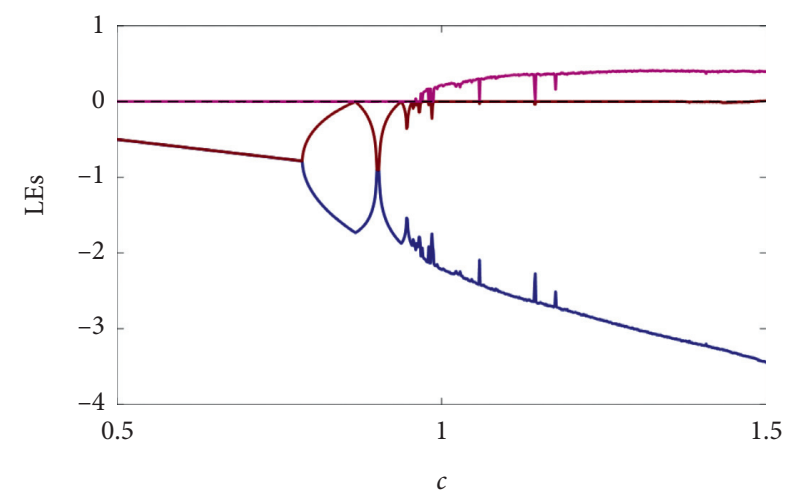

(d)

Figure 2: (a) The bifurcation diagram and (b) Lyapunov exponents of system (1) concerning the changes of parameter $b \in[0.7,2.5]$. (c) The bifurcation diagram and (d) the Lyapunov exponents of system (1) concerning the changes of parameter $c \in[0.5,1.5]$. The value of parameter $a$ is considered to be equal to 2 with the initial condition of $\left(\rho_{0}, \theta_{0}, \varphi_{0}\right)=(-2,3,0)$. The occurrence of chaos behavior is justified based on the bifurcation diagrams and Lyapunov exponents.

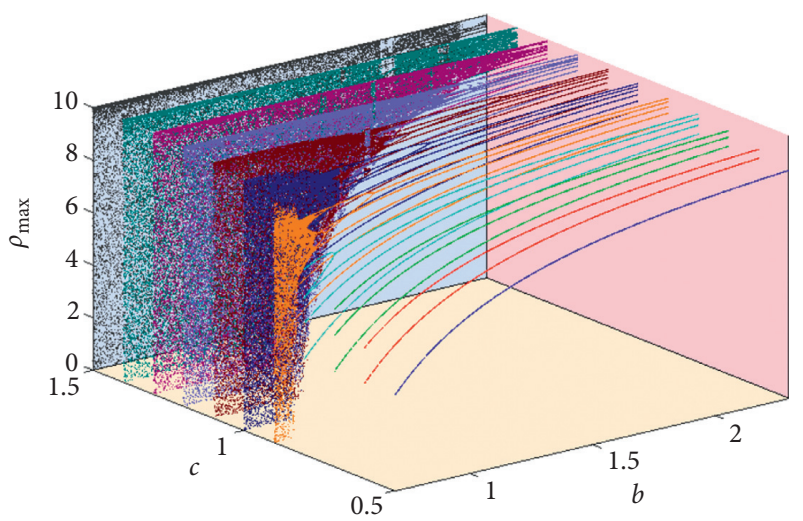

(a)

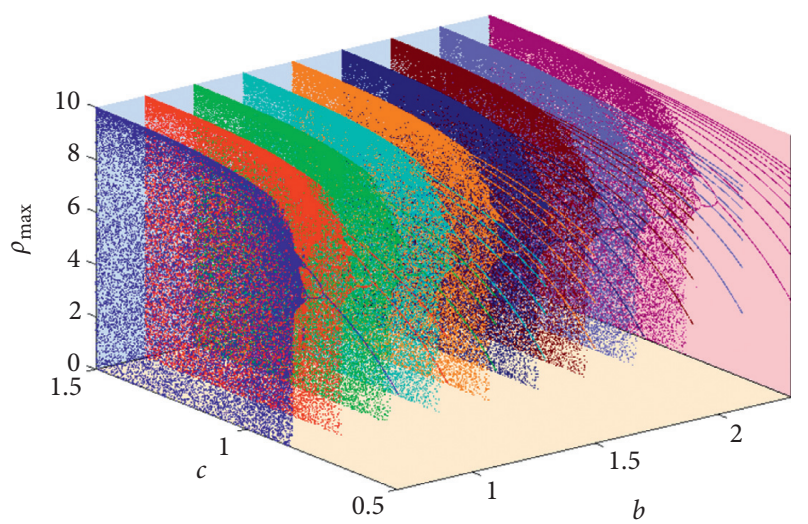

(b)

Figure 3: Two 3D bifurcation diagrams of system (1) concerning the changes of parameter $b$ and $c$ with the different horizontal resolution by assuming $a=2$ and initial condition of $\left(\rho_{0}, \theta_{0}, \varphi_{0}\right)=(-2,3,0)$. (a) Eleven bifurcation diagrams corresponding to the variation of $b$ parameter and eleven values of $c$ parameter. (b) Nine bifurcation diagrams corresponding to the variation of $c$ parameter and nine values of $b$ parameter. Dynamic changes of the system are noticeable for simultaneous changes of $b$ and $c$ parameters.

the basin of attraction can help to select proper initial conditions for a system to exhibit chaos. Figure 4 shows two $2 \mathrm{D}$ basins of attraction of the introduced system in the $\rho-\theta$ plane with the $\varphi_{1}=2$ and $\varphi_{2}=0$. The initial value for $\varphi$ variable is selected in a way that the system's equilibria can be depicted in each basin of attraction. As represented in Figure $4(\mathrm{a})$, the second equilibrium of the system, i.e., $\left(\rho_{2}^{*}, \theta_{2}^{*}, \varphi_{2}^{*}\right)=(8,2,2)$, can be observed within the attractor's basin of attraction. Also, the first equilibrium $\left(\rho_{1}^{*}, \theta_{1}^{*}, \varphi_{1}^{*}\right)=$ $(10,0,0)$ is located on the sphere in Figure $4(b)$. Since the 


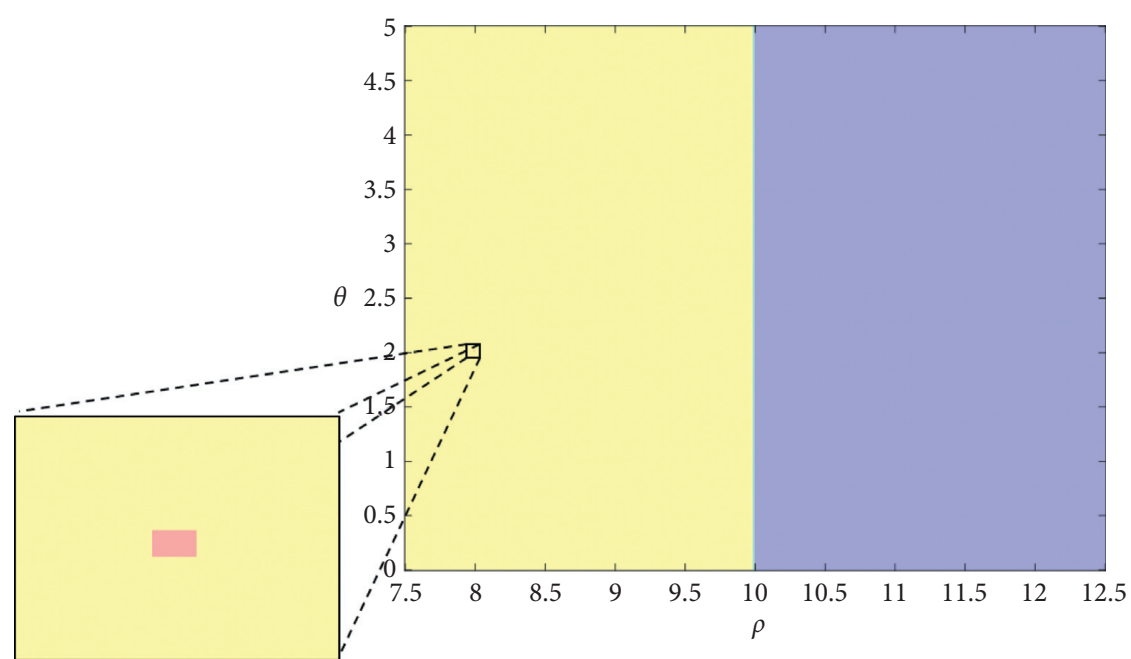

(a)

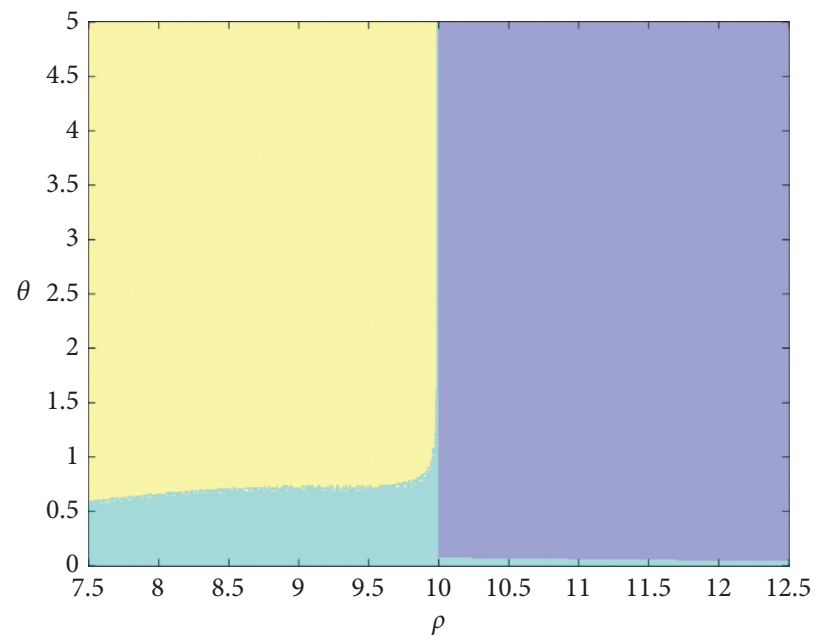

(b)

FIgURE 4: Basin of attraction of system (1) with $a=2, b=1$, and $c=1$ in the $\rho$ - $\theta$ plane with the fixed initial value of (a) $\varphi=2$ and (b) $\varphi=0$. All initial values of yellow color lead to the strange attractor of the system, and the purple ones lead to the unbounded orbits. The point which is shown in pink color leads to the equilibrium $\left(\rho_{2}^{*}, \theta_{2}^{*}, \varphi_{2}^{*}\right)=(8,2,2)$. The points represented in cyan color remain on the sphere of radius 10 . Each unstable equilibria of the system can be seen in each represented basin of attraction. This helps to decide whether the attractor is selfexcited or hidden.

unstable equilibrium $\left(\rho_{2}^{*}, \theta_{2}^{*}, \varphi_{2}^{*}\right)=(8,2,2)$ is in the chaotic basin of attraction, it can be expressed that the proposed system owns a self-excited strange attractor. Moreover, as illustrated in Figure 4, the sphere owns a basin of attraction, which means that there are some initial conditions that are attracted to the sphere, not to the strange attractor.

It is worth mentioning that the basins of attraction demonstrated in Figure 4 are obtained by identifying different system's behavior and specifying a distinctive color to each attractor. More generally, the infinite unstable behavior, which crosses a threshold, is identified in the first step. If the system behavior is constant, it will be investigated whether it is a fixed point or not. The next step is to calculate the period of the signal. If we can find a finite period for a signal, we will label the attractor as a periodic attractor. Otherwise, we will determine it as a strange attractor. After identifying the system's attractors, the system should be run for a range of initial conditions, and the ultimate attractor they reach should be marked with a specific color of that attractor.

2.1. Connecting Curves. Vortex core curves, known as connecting curves, were first studied by Roth and Peikert [45] and then were widely investigated by Gilmore et al. [46]. Connecting curves are 1D sets that provide further information about the system's dynamics compared to equilibria, defined as $0 \mathrm{D}$ sets. While the fixed points provide limited information about the system's trajectory, connecting curves provide extensive information about it. In other words, fixed points only provide local information about the nature of the flows. On the contrary, connecting curves can help in obtaining more general information 


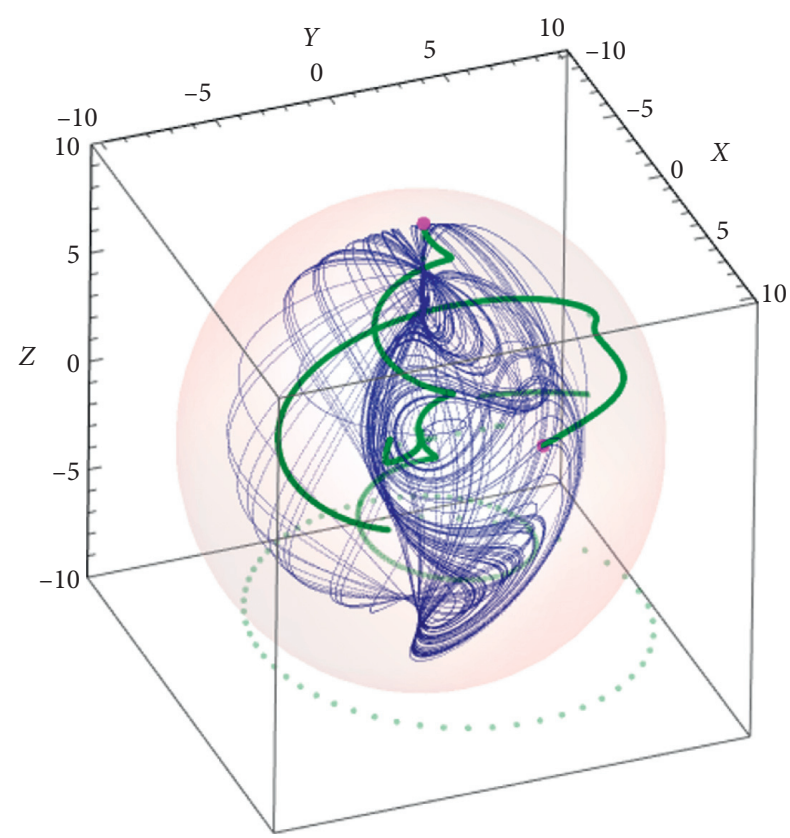

FIgUre 5: The 3D strange attractor of system (1) in Cartesian coordinates along with its connecting curves, which are shown in green, considering variable $\varphi$ as the phase space coordinate. The two unstable equilibria of system $(1)$, i.e., $\left(\rho_{1}^{*}, \theta_{1}^{*}, \varphi_{1}^{*}\right)=(10,0,0)$ and $\left(\rho_{2}^{*}, \theta_{2}^{*}, \varphi_{2}^{*}\right)=(8,2,2)$ are represented in magenta color. Connecting curves of the system connect the equilibria of the system and approximately define how trajectory changes.

about the flow's nature and the system's trajectory. So, they might be promising to identify chaotic system dynamics. According to [46], connecting curves can be obtained based on the system's dynamics and geometric theories. Based on the system's dynamics, connecting curves are the curves around which the direction of velocity and acceleration are the same. Similarly, based on geometric theories, connecting curves are zero curvatures which mean that the cross product of velocity and acceleration must be zero. Both approaches would lead to the same concept, which is connecting curves. Moreover, connecting curves should necessarily cross all the equilibria of the system; however, they are not dependent on the equilibria's existence. In fact, connecting curves connect the fixed points of a system in a meaningful way, guiding how trajectory alters. Based on the system's dynamic, which refers to the second method proposed in [46], the connecting curves of any systems can be obtained from the points satisfying the following condition:

$$
J \vec{V}-\lambda \vec{V}=0
$$

Here, $\vec{V}$ denotes the velocity vector consisting of the velocity of each phase space variable. Also, $J$ and $\lambda$ refer to the system's Jacobian matrix and its eigenvalues, respectively. For an $\mathrm{nD}$ system, using equation (4), $n$ equations can be obtained with $n+1$ variables: phase space variables plus eigenvalue $\lambda .1 D$ set can be obtained by considering a primary phase space variable and obtaining other variables in terms of the selected primary variable and $\lambda$ using $n$ equations.

In order to implement the connecting curves of system (1), first, three main equations with four variables, namely, $\rho$, $\theta, \varphi$, and $\lambda$, are obtained using equation (4). Then, considering the variable $\varphi$ as the primary phase space variable, two other variables, i.e., $\rho$ and $\theta$ can be written down in terms of $\varphi$ and $\lambda: \rho(\varphi, \lambda)$ and $\theta(\varphi, \lambda)$. Next, $\lambda$ is written down in terms of $\varphi: \lambda(\varphi)$. The solution of $\lambda(\varphi)=0$ leads to 5 roots for each value of $\varphi$. Obtaining the real eigenvalues for each value of $\varphi$, other variables can be easily calculated, and the connecting curves of the system can be finally plotted. The implementation of connecting curves for system (1) in Cartesian coordinates is illustrated in Figure 5. According to Figure 5, it can be seen that the connecting curves pass through the two unstable equilibria of the system, i.e., $\left(\rho_{1}^{*}, \theta_{1}^{*}, \varphi_{1}^{*}\right)=(10,0,0)$ and $\left(\rho_{2}^{*}, \theta_{2}^{*}, \varphi_{2}^{*}\right)=(8,2,2)$. Since connecting curves are calculated based on the system's dynamics, they can have a nonuniform resolution. As a result, The separate points in Figure 5 are also connecting curves of the proposed system.

\section{Conclusion}

In this paper, a new chaotic system defined in the spherical coordinates was presented. The dynamic properties of the proposed system were investigated using the $1 \mathrm{D}$ and $2 \mathrm{D}$ bifurcation diagrams and also the Lyapunov exponents. Both bifurcation diagrams and Lyapunov exponents confirmed the existence of chaos in the introduced system. Moreover, the inverse period-doubling route to chaos and perioddoubling route to the chaos was observed in the system's bifurcation for some parameters' values. The initial condition for plotting the system's attractor, bifurcation diagrams, and Lyapunov exponents was considered the same and was obtained using a comprehensive search. Furthermore, the initial condition was considered constant for each bifurcation parameter to plot the system's bifurcation diagrams. It was found that the proposed system owns two equilibria. Stability analysis of the system's equilibria demonstrated that both system's equilibria are unstable. Besides, investigation of the system's basin of attraction specified the regions where the initial values led to the strange attractor or the sphere. In order to investigate whether the strange attractor is hidden or not, two basins of attraction with the initial $\varphi$ values equal to the $\varphi$ values of the system's equilibria were plotted. It turned out that the introduced system owns a self-excited strange attractor, which is captivated in an insurmountable sphere since one unstable equilibrium was observed in the basin of attraction of the strange attractor. So, when the system solution touches the sphere, it remains on it. Ultimately, the connecting curves of the proposed system were implemented using a parameterized method to provide general information about the system's trajectory. Generally, investigating a new chaotic system with a typical feature helps to understand chaos better and define systems that can lead to this comprehension. The proposed system can be used in some applications, such as image encryption or generating random numbers in future works. 


\section{Data Availability}

The data used to support the findings of the study are available within the article.

\section{Conflicts of Interest}

The authors declare that they have no conflicts of interest.

\section{Acknowledgments}

The authors extend their gratitude to the Deanship of Scientific Research at King Khalid University for funding this work through research group program under Grant no. R. G. P. $2 / 48 / 42$.

\section{References}

[1] J. Kengne, A. N. Negou, and D. Tchiotsop, "Antimonotonicity, chaos and multiple attractors in a novel autonomous memristor-based jerk circuit," Nonlinear Dynamics, vol. 88, no. 4, pp. 2589-2608, 2017.

[2] J. C. Sprott, "Some simple chaotic flows," Physical Review E, vol. 50, no. 2, pp. R647-R650, 1994.

[3] D. Ghosh, A. R. Chowdhury, and P. Saha, "Multiple delay Rössler system-Bifurcation and chaos control," Chaos, Solitons \& Fractals, vol. 35, no. 3, pp. 472-485, 2008.

[4] M. Sayeed Anwar, G. K. Sar, A. Ray, and D. Ghosh, "Behavioral study of a new chaotic system," The European Physical Journal Special Topics, vol. 229, no. 6-7, pp. 13431350, 2020.

[5] A. Ray and D. Ghosh, "Another new chaotic system: bifurcation and chaos control," International Journal of Bifurcation and Chaos, vol. 30, no. 11, Article ID 2050161, 2020.

[6] H. Bao, Z. Hua, N. Wang, L. Zhu, M. Chen, and B. Bao, "Initials-boosted coexisting chaos in a 2-D sine map and its hardware implementation," IEEE Transactions on Industrial Informatics, vol. 17, no. 2, pp. 1132-1140, 2020.

[7] B. Bao, Y. Zhu, C. Li, H. Bao, and Q. Xu, "Global multistability and analog circuit implementation of an adapting synapsebased neuron model," Nonlinear Dynamics, vol. 101, no. 2, pp. 1105-1118, 2020.

[8] A. Ray, D. Ghosh, and A. R. Chowdhury, "Topological study of multiple coexisting attractors in a nonlinear system," Journal of Physics A: Mathematical and Theoretical, vol. 42, no. 38, Article ID 385102, 2009.

[9] A. Bayani, K. Rajagopal, A. J. M. Khalaf, S. Jafari, G. D. Leutcho, and J. Kengne, "Dynamical analysis of a new multistable chaotic system with hidden attractor: antimonotonicity, coexisting multiple attractors, and offset boosting," Physics Letters A, vol. 383, no. 13, pp. 1450-1456, 2019.

[10] J. C. Sprott, S. Jafari, A. J. M. Khalaf, and T. Kapitaniak, "Megastability: coexistence of a countable infinity of nested attractors in a periodically-forced oscillator with spatiallyperiodic damping," The European Physical Journal Special Topics, vol. 226, no. 9, pp. 1979-1985, 2017.

[11] G. D. Leutcho, A. J. M. Khalaf, Z. Njitacke Tabekoueng et al., "A new oscillator with mega-stability and its Hamilton energy: infinite coexisting hidden and self-excited attractors," Chaos: An Interdisciplinary Journal of Nonlinear Science, vol. 30, no. 3, Article ID 033112, 2020.
[12] G. D. Leutcho, S. Jafari, I. I. Hamarash, J. Kengne, Z. Tabekoueng Njitacke, and I. Hussain, "A new megastable nonlinear oscillator with infinite attractors," Chaos, Solitons \& Fractals, vol. 134, Article ID 109703, 2020.

[13] Y. Zhang, Z. Liu, H. Wu, S. Chen, and B. Bao, "Two-memristor-based chaotic system and its extreme multistability reconstitution via dimensionality reduction analysis," Chaos, Solitons \& Fractals, vol. 127, pp. 354-363, 2019.

[14] Y. Zhang, Z. Liu, H. Wu, S. Chen, and B. Bao, "Extreme multistability in memristive hyper-jerk system and stability mechanism analysis using dimensionality reduction model," The European Physical Journal Special Topics, vol. 228, no. 10, pp. 1995-2009, 2019.

[15] H. Li, H. Bao, L. Zhu, B. Bao, and M. Chen, "Extreme multistability in simple area-preserving map," IEEE Access, vol. 8, pp. 175972-175980, 2020.

[16] C. Li and J. C. Sprott, "Variable-boostable chaotic flows," Optik, vol. 127, no. 22, pp. 10389-10398, 2016.

[17] V.-T. Pham, A. Akgul, C. Volos, S. Jafari, and T. Kapitaniak, "Dynamics and circuit realization of a no-equilibrium chaotic system with a boostable variable," AEU - International Journal of Electronics and Communications, vol. 78, pp. 134-140, 2017.

[18] R. Zhang, A. Wu, S. Zhang, Z. Wang, and S. Cang, "Dynamical analysis and circuit implementation of a DC/DC single-stage boost converter with memristance load," Nonlinear Dynamics, vol. 93, no. 3, pp. 1741-1755, 2018.

[19] H. Bao, Y. Zhang, W. Liu, and B. Bao, "Memristor synapsecoupled memristive neuron network: synchronization transition and occurrence of chimera," Nonlinear Dynamics, vol. 100, no. 1, pp. 937-950, 2020.

[20] M. Chen, M. Sun, H. Bao, Y. Hu, and B. Bao, "Flux-charge analysis of two-memristor-based chua's circuit: dimensionality decreasing model for detecting extreme multistability," IEEE Transactions on Industrial Electronics, vol. 67, no. 3, pp. 2197-2206, 2019.

[21] S. Cang, Z. Kang, and Z. Wang, "Pseudo-random number generator based on a generalized conservative Sprott-A system," Nonlinear Dynamics, vol. 104, no. 1, pp. 827-844, 2021.

[22] Y. Li, S. Cang, Z. Kang, and Z. Wang, "A new conservative system with isolated invariant tori and six-cluster chaotic flows," The European Physical Journal Special Topics, vol. 229, no. 6-7, pp. 1335-1342, 2020.

[23] S. Cang, Y. Li, W. Xue, Z. Wang, and Z. Chen, "Conservative chaos and invariant tori in the modified Sprott A system," Nonlinear Dynamics, vol. 99, no. 2, pp. 1699-1708, 2020.

[24] S. Cang, Y. Li, Z. Kang, and Z. Wang, "Generating multicluster conservative chaotic flows from a generalized Sprott-A system," Chaos, Solitons \& Fractals, vol. 133, Article ID 109651, 2020.

[25] C. Li, J. C. Sprott, Y. Liu, Z. Gu, and J. Zhang, "Offset boosting for breeding conditional symmetry," International Journal of Bifurcation and Chaos, vol. 28, no. 14, Article ID 1850163, 2018.

[26] C. Li, Y. Xu, G. Chen, Y. Liu, and J. Zheng, "Conditional symmetry: bond for attractor growing," Nonlinear Dynamics, vol. 95, no. 2, pp. 1245-1256, 2019.

[27] C. Li, J. C. Sprott, and H. Xing, "Constructing chaotic systems with conditional symmetry," Nonlinear Dynamics, vol. 87, no. 2, pp. 1351-1358, 2017

[28] G. D. Leutcho and J. Kengne, "A unique chaotic snap system with a smoothly adjustable symmetry and nonlinearity: chaos, offset-boosting, antimonotonicity, and coexisting multiple attractors," Chaos, Solitons \& Fractals, vol. 113, pp. 275-293, 2018 . 
[29] V.-T. Pham, S. Vaidyanathan, and T. Kapitaniak, "Complexity, dynamics, control, and applications of nonlinear systems with multistability," Complexity, vol. 2020, Article ID 8510930, 2020.

[30] J. Ruan, K. Sun, J. Mou, S. He, and L. Zhang, "Fractional-order simplest memristor-based chaotic circuit with new derivative," The European Physical Journal Plus, vol. 133, no. 1, p. 3, 2018.

[31] S. Jafari, J. C. Sprott, and S. M. R. Hashemi Golpayegani, "Elementary quadratic chaotic flows with no equilibria," Physics Letters A, vol. 377, no. 9, pp. 699-702, 2013.

[32] M. Molaie, S. Jafari, J. C. Sprott, and S. M. R. H. Golpayegani, "Simple chaotic flows with one stable equilibrium," International Journal of Bifurcation and Chaos, vol. 23, no. 11, Article ID 1350188, 2013.

[33] S. Ren, S. Panahi, K. Rajagopal, A. Akgul, V.-T. Pham, and S. Jafari, "A new chaotic flow with hidden attractor: the first hyperjerk system with No equilibrium," Zeitschrift für Naturforschung A, vol. 73, no. 3, pp. 239-249, 2018.

[34] C. Wang, C. Hu, J. Han, and S. Cang, "A new no-equilibrium chaotic system and its topological horseshoe chaos," Advances in Mathematical Physics, vol. 2016, Article ID 3142068, 6 pages, 2016.

[35] S. Nag Chowdhury and D. Ghosh, "Hidden attractors: a new chaotic system without equilibria," The European Physical Journal Special Topics, vol. 229, no. 6-7, pp. 1299-1308, 2020.

[36] Z. Wei, J. C. Sprott, and H. Chen, "Elementary quadratic chaotic flows with a single non-hyperbolic equilibrium," Physics Letters A, vol. 379, no. 37, pp. 2184-2187, 2015.

[37] C.-L. Li and J.-B. Xiong, "A simple chaotic system with nonhyperbolic equilibria," Optik, vol. 128, pp. 42-49, 2017.

[38] X. Wang and G. Chen, "A chaotic system with only one stable equilibrium," Communications in Nonlinear Science and Numerical Simulation, vol. 17, no. 3, pp. 1264-1272, 2012.

[39] V.-T. Pham, S. Jafari, T. Kapitaniak, C. Volos, and S. T. Kingni, "Generating a chaotic system with one stable equilibrium," International Journal of Bifurcation and Chaos, vol. 27, no. 4, Article ID 1750053, 2017.

[40] S. Jafari and J. C. Sprott, "Simple chaotic flows with a line equilibrium," Chaos, Solitons \& Fractals, vol. 57, pp. 79-84, 2013.

[41] T. Gotthans and J. Petržela, "New class of chaotic systems with circular equilibrium," Nonlinear Dynamics, vol. 81, no. 3, pp. 1143-1149, 2015.

[42] S. Panahi, Z. Aram, S. Jafari, V.-T. Pham, C. Volos, and K. Rajagopal, "A new transiently chaotic flow with ellipsoid equilibria," Pramana, vol. 90, no. 3, p. 31, 2018.

[43] S. Jafari, J. C. Sprott, and M. Molaie, "A simple chaotic flow with a plane of equilibria," International Journal of Bifurcation and Chaos, vol. 26, no. 6, Article ID 1650098, 2016.

[44] S. Jafari, J. C. Sprott, V.-T. Pham, C. Volos, and C. Li, "Simple chaotic 3D flows with surfaces of equilibria," Nonlinear Dynamics, vol. 86, no. 2, pp. 1349-1358, 2016.

[45] M. Roth and R. Peikert, "A higher-order method for finding vortex core lines," in Proceedings Visualization'98 (Cat. No. 98CB36276), pp. 143-150, IEEE, Research Triangle Park, NC, USA, October 1998.

[46] R. Gilmore, J.-M. Ginoux, T. Jones, C. Letellier, and U. S. Freitas, "Connecting curves for dynamical systems," Journal of Physics A: Mathematical and Theoretical, vol. 43, no. 25, Article ID 255101, 2010.

[47] J. C. Sprott, Elegant Chaos: Algebraically Simple Chaotic Flows, World Scientific, Singapore, 2010.
[48] Z. Wei, V.-T. Pham, T. Kapitaniak, and Z. Wang, "Bifurcation analysis and circuit realization for multiple-delayed WangChen system with hidden chaotic attractors," Nonlinear Dynamics, vol. 85, no. 3, pp. 1635-1650, 2016.

[49] S. Jafari and J. C. Sprott, "Simple chaotic flows with a line equilibrium," Chaos, Solitons \& Fractals, vol. 57, pp. 341-342, 2015.

[50] S. Cang, Y. Li, R. Zhang, and Z. Wang, "Hidden and selfexcited coexisting attractors in a Lorenz-like system with two equilibrium points," Nonlinear Dynamics, vol. 95, no. 1, pp. 381-390, 2019.

[51] M. Chen, C. Wang, H. Bao, X. Ren, B. Bao, and Q. Xu, "Reconstitution for interpreting hidden dynamics with stable equilibrium point," Chaos, Solitons \& Fractals, vol. 140, Article ID 110188, 2020.

[52] M.-F. Danca, N. V. Kuznetsov, and G. Chen, "Approximating hidden chaotic attractors via parameter switching," Chaos: An Interdisciplinary Journal of Nonlinear Science, vol. 28, no. 1, Article ID 013127, 2018.

[53] M.-F. Danca, P. Bourke, and N. Kuznetsov, "Graphical structure of attraction basins of hidden chaotic attractors: the Rabinovich-Fabrikant system," International Journal of Bifurcation and Chaos, vol. 29, no. 1, Article ID 1930001, 2019.

[54] M.-F. Danca and M. Fečkan, "Hidden chaotic attractors and chaos suppression in an impulsive discrete economical supply and demand dynamical system," Communications in Nonlinear Science and Numerical Simulation, vol. 74, pp. 1-13, 2019.

[55] G. A. Leonov, N. V. Kuznetsov, and T. N. Mokaev, "Homoclinic orbits, and self-excited and hidden attractors in a Lorenz-like system describing convective fluid motion," The European Physical Journal Special Topics, vol. 224, no. 8, pp. 1421-1458, 2015, in English.

[56] N. V. Kuznetsov and G. A. Leonov, "Hidden attractors in dynamical systems: systems with no equilibria, multistability and coexisting attractors," IFAC Proceedings Volumes, vol. 47, no. 3, pp. 5445-5454, 2014.

[57] S. Jafari, S. Dehghan, G. Chen, S. T. Kingni, and K. Rajagopal, "Twin birds inside and outside the cage," Chaos, Solitons \& Fractals, vol. 112, pp. 135-140, 2018.

[58] F. Nazarimehr, V.-T. Pham, K. Rajagopal, F. E. Alsaadi, T. Hayat, and S. Jafari, "A new imprisoned strange attractor," International Journal of Bifurcation and Chaos, vol. 29, no. 13, Article ID 1950181, 2019.

[59] L. Chen, E. Tlelo-Cuautle, I. I. Hamarash, V.-T. Pham, and H. R. Abdolmohammadi, "A novel chaotic system in the spherical coordinates," The European Physical Journal Special Topics, vol. 229, no. 6-7, pp. 1257-1263, 2020.

[60] A. Wolf, J. B. Swift, H. L. Swinney, and J. A. Vastano, "Determining Lyapunov exponents from a time series," Physica D: Nonlinear Phenomena, vol. 16, no. 3, pp. 285-317, 1985. 